\title{
Investigation of TTF Injector Alignment with the Simulation Code $\mathrm{V}^{*}$
}

\author{
W. Beinhauer, R. Cee ${ }^{\ddagger}$, W. Koch, M. Krassilnikov, A. Novokhatski ${ }^{\S}$, \\ S. Ratschow, T. Weiland, Darmstadt University of Technology, FB18, Germany \\ P. Castro, S. Schreiber, DESY, Hamburg, Germany
}

\begin{abstract}
The exact alignment of accelerator components is of crucial importance for the production of low emittance beams. Once a beam-line section is set up, a supplementary correction of misalignments implies the knowledge of its magnitude which is difficult to determine using conventional adjusting instruments. An excellent alternative to measure existing misalignments of accelerator components is to vary machine parameters and compare the behaviour of the beam with results obtained from a simulation. It is obvious that time consuming particle tracking programmes are not appropriate to reach this aim. Regarding computing time, the on-line simulation code $\mathrm{V}$ is advantageous compared to other beam dynamics programmes. The theoretical basis of V-Code, the "Ensemble Model", consists of selfconsistent equations for the ensemble parameters that are derived from the Vlasov equation. The requirement to simulate misalignments such as offsets and tilts led to the development of the ALIGNMENT UTILITY which utilizes the solver of V-Code. The new utility enabled us to investigate the beam-line alignment of the TESLA Test Facility injector. This contribution presents the theoretical background and an illustrating example of the optimization process.
\end{abstract}

\section{INTRODUCTION}

Most of the currently available beam dynamics programmes apply single particle dynamics on a set of "macro particles" defined by their position in phase space and their charge-to-mass ratio. The accuracy of this method depends on the number of macro particles used for the simulation. As the simulation time increases rapidly with this number such programmes are not applicable if a sequence of simulations has to be carried out or if the simulation has to be on-line. This limitation cease to exist if the Vlasov equation is applied to particle beams as it is done in the online beam dynamics programme V-Code [1]. The theoretical basis of the V-Code solver is the "Ensemble Model" which treats the beam as an ensemble of particles that is not only described by its position and momentum but also by the correlations in the six dimensional phase space [2]. In the approximation of linear forces the phase distribution function can be replaced by a set of ensemble parameters. The time evolution of these parameters results from a system of coupled differential equations that are derived from the Vlasov equation. The ALIGnMent UtiLity uses

\footnotetext{
${ }^{*}$ Work supported in part by DESY, Germany and DFG (FOR 272/2-1).

$\ddagger$ cee@temf.de

$\S$ Now at SLAC, USA.
}

the V-Code solver for the detection of misalignments of accelerator components by optimizing the misalignment parameters until simulation and measured data yield a good agreement.

\section{THE ENSEMBLE MODEL}

To simplify the formulation of the Ensemble Model let us first introduce some notations. For the particle's velocity $\vec{V}$, energy $E$ and momentum $\vec{P}$ we write the normalized quantities

$$
\vec{\beta}=\frac{\vec{V}}{c}, \quad \gamma=\frac{E}{m c^{2}} \quad \text { and } \quad \vec{p}=\frac{\vec{P}}{m c},
$$

with the speed of light $c$ and the particle mass $m$. In the time equations of subsection 2.2 we will use $\tau=c \cdot t$ as time coordinate and $\overrightarrow{\mathbf{F}}=\vec{F} / m c^{2}$ as normalized force.

\subsection{Ensemble Parameters}

An ensemble is described by six first order and $21 \mathrm{sec}$ ond order parameters. The first order parameters are the average values of the particles' positions and momenta and can be grouped in a vector $\langle\vec{R}\rangle$ :

$$
\langle\vec{R}\rangle=(\langle\vec{r}\rangle,\langle\vec{p}\rangle)=\left(\langle x\rangle,\langle y\rangle,\langle z\rangle,\left\langle p_{x}\right\rangle,\left\langle p_{y}\right\rangle,\left\langle p_{z}\right\rangle\right) .
$$

One can further define a vector $\Delta \vec{R}=\vec{R}-\langle\vec{R}\rangle$ as a measure for the variation of a particle from the average values. The second order moments, representing the correlations between pairs of these variations, can be expressed by

$$
M_{i j}=\left\langle\Delta R_{i} \Delta R_{j}\right\rangle \text { for } i, j=1,2, \ldots 6 .
$$

Arranging the second order moments into three separate $3 \times 3$ matrices we get for $i, j=1,2,3$ :

1. Matrix $\widehat{\mathbf{S}}$ for the position-position correlations:

$$
\widehat{\mathbf{S}}_{i j}=M_{i j}
$$

2. Matrix $\widehat{\mathbf{L}}$ for the position-momentum correlations:

$$
\widehat{\mathbf{L}}_{i j}=M_{i(j+3)}
$$

3. Matrix $\widehat{\mathbf{T}}$ for the momentum-momentum correlations:

$$
\widehat{\mathbf{T}}_{i j}=M_{(i+3)(j+3)}
$$

Due to the relation $M_{i j}=M_{j i}$ (from (1)) the matrices $\widehat{\mathbf{S}}$ and $\widehat{\mathbf{T}}$ are symmetric whereas matrix $\widehat{\mathbf{L}}$ is not. The three matrices can be combined to a single one:

$$
\widehat{\mathbf{M}}=\left(\begin{array}{cc}
\widehat{\mathbf{S}} & \widehat{\mathbf{L}} \\
\widehat{\mathbf{L}}^{T} & \widehat{\mathbf{T}}
\end{array}\right)
$$


The determinant of $\widehat{\mathbf{M}}$ is the square of the volume in the six-dimensional phase space occupied by the ensemble and thus the square of the total beam emittance $\varepsilon$ :

$$
\varepsilon=\operatorname{det}(\widehat{\mathbf{M}})^{-1 / 2} \text {. }
$$

To clarify the meaning of the ensemble parameters for an uncoupled motion one can correlate them with the well known Courant-Snyder parameters. Projecting the phase space on the the $\mathrm{x}-\mathrm{p}_{\mathrm{x}}$-plane the following relations between the Courant-Snyder parameters, denoted with $\alpha_{x}, \beta_{x}$ and $\gamma_{x}$, and the correlations from the Ensemble Model hold:

$$
\alpha_{x}=-\frac{L_{11}}{\varepsilon}, \quad \beta_{x}=\frac{S_{11}}{\varepsilon}, \quad \gamma_{x}=\frac{T_{11}}{\varepsilon},
$$

where $\varepsilon$ is the full emittance given by equation (2).

\subsection{Set of time equations}

Within the scope of the Ensemble Model the behaviour of the beam in time is described by a set of 27 coupled ordinary differential equations, expressing the time dependence of each ensemble parameter. These equations are derived from Vlasov equation for vanishing dissipative forces,

$\frac{\partial \psi(t, \vec{r}, \vec{p})}{\partial t}+\nabla_{r} \psi(t, \vec{r}, \vec{p}) \cdot \frac{\vec{p}}{\gamma} c+\nabla_{p} \psi(t, \vec{r}, \vec{p}) \cdot \frac{\vec{F}}{m c}=0$,

stating that the volume occupied by a given number of particles in phase space remains constant. Multiplying equation (3) by $\mu / c$, where $\langle\mu\rangle$ may be any of the ensemble parameters, and applying a partial integration yields the equation for average values:

$$
-\frac{\partial\langle\mu\rangle}{\partial \tau}+\left\langle\nabla_{r} \mu \cdot \frac{\vec{p}}{\gamma}\right\rangle+\left\langle\nabla_{p} \mu \cdot \overrightarrow{\mathbf{F}}\right\rangle=0 .
$$

The averaging of the second term necessitates the substitution of the factor $1 / \gamma$ by a Taylor expansion:

$$
\frac{1}{\gamma}=\frac{1}{\sqrt{1+\vec{p}^{2}}} \approx \frac{1}{\gamma_{m}}-\frac{1}{2 \gamma_{m}^{3}} \sum_{i=1}^{3}\left(2\left\langle p_{i}\right\rangle \Delta p_{i}^{2}-\widehat{\mathbf{T}}_{i i}\right)
$$

where terms of the order $\Delta \vec{p}^{2}=(\vec{p}-\langle\vec{p}\rangle)^{2}$ are neglected, which is only valid if $\Delta \vec{p} \ll\langle\vec{p}\rangle$, i.e. if the energy distribution of the beam is sharp enough. In equation (4) $\gamma_{m}$ denotes the root-mean-square of the particle energy $\gamma$. The time equations result from the application of equation (3) to each ensemble parameter [2]. They can be expressed in matrix form:

1. Average position:

$$
\frac{\partial\langle\vec{r}\rangle}{\partial \tau}=\widehat{\mathbf{W}} \cdot\langle\vec{p}\rangle, \text { where } \widehat{\mathbf{W}}_{i j}=\frac{\delta_{i j}}{\gamma_{m}}-\frac{\widehat{\mathbf{T}}_{i j}}{\gamma_{m}^{3}}
$$

2. Average momentum:

$$
\frac{\partial\langle\vec{p}\rangle}{\partial \tau}=\overrightarrow{\mathbf{F}}
$$

3. Position-position correlations:

$$
\frac{\partial \widehat{\mathbf{S}}}{\partial \tau}=\widehat{\mathbf{V}} \cdot \widehat{\mathbf{L}}^{T}+\widehat{\mathbf{L}} \cdot \widehat{\mathbf{V}}, \text { where } \widehat{\mathbf{V}}_{i j}=\frac{\delta_{i j}}{\gamma_{m}}-\frac{\left\langle p_{i}\right\rangle\left\langle p_{j}\right\rangle}{\gamma_{m}^{3}}
$$

4. Position-momentum correlations:

$$
\frac{\partial \widehat{\mathbf{L}}}{\partial \tau}=\widehat{\mathbf{V}} \cdot \widehat{\mathbf{T}}+\widehat{\mathbf{Y}}, \text { where } \widehat{\mathbf{Y}}_{i j}=\left\langle\left(r_{i}-\left\langle r_{i}\right\rangle\right) \mathbf{F}_{j}\right\rangle
$$

5. Momentum-momentum correlations:

$$
\frac{\partial \widehat{\mathbf{T}}}{\partial \tau}=\widehat{\mathbf{Z}}+\widehat{\mathbf{Z}}^{T}, \text { where } \widehat{\mathbf{Z}}_{i j}=\left\langle\left(p_{i}-\left\langle p_{i}\right\rangle\right) \mathbf{F}_{j}\right\rangle
$$

If one deals with linear forces a Taylor expansion can be applied to $\overrightarrow{\mathbf{F}}$ allowing the solution of the averaging expressions in the matrices $\widehat{\mathbf{Y}}$ and $\widehat{\mathbf{Z}}$ [3]. The subsequent reformulation of the matrix equations forms the basis for a computational evaluation [4].

\section{THE ALIGNMENT UTILITY}

The ALIGNMENT UTILITY is a PC based MDI windows application which treats beam dynamic calculations with the simulation Code V in combination with data acquired by means of an experiment. It is capable to deliver a set of alignment parameters which brings the simulation into agreement with the corresponding data. The measured quantity serving for comparison is the beam position at a certain location of the beam-line which is provided with a suitable measuring device. The optimization process itself is based on the minimization of a functional $\phi(\vec{u})$ which depends on a set of $l$ alignment parameters $u_{1}, \ldots, u_{l}$ summarized in a vector $\vec{u}$. For a single series of measurements it has the following form:

$$
\begin{aligned}
\phi(\vec{u}) & =\sum_{i=1}^{n}\left[w_{i}^{x}\left(x_{i}^{\text {sim }}(\vec{u})-x_{i}^{\text {meas }}(\vec{u})\right)^{2}+\right. \\
& \left.+w_{i}^{y}\left(y_{i}^{\text {sim }}(\vec{u})-y_{i}^{\text {meas }}(\vec{u})\right)^{2}\right]+f_{p}\left(\vec{u}, \vec{u}^{\text {min }}, \vec{u}^{\text {max }}\right)
\end{aligned}
$$

where $n$ denotes the number of data points of the series of measurements, $w_{i}^{x}, w_{i}^{y}$ are weight factors of data point $i$ which are connected with the data's error and $x_{i}^{\text {sim }}, y_{i}^{\text {sim }}$ respectively $x_{i}^{\text {meas }}, y_{i}^{\text {meas }}$ are the simulated and measured $\mathrm{x}-$ and $\mathrm{y}$-positions of the beam. The function $f_{p}\left(\vec{u}, \vec{u}^{\min }, \vec{u}^{\max }\right)$ is a penalty function which increases rapidly when the alignment parameters $\vec{u}$ found in an intermediate step of the optimization approach the user defined limits $\vec{u}^{\text {min }}$ and $\vec{u}^{\max }$. As the optimization is searching for the minimum of the functional this ensures the result to remain within a predefined range. It is also possible to synchronously optimize several series of measurements. In this case the functional to be evaluated is the sum over all functionals $\phi(\vec{u})$ which ought to be considered. To yield the value of the functional the V-Code solver is employed. It simulates the beam until the location of the measurement device using the current settings 
of the machine and returns the beam position. At the current status of the development the one-dimensional $(l=1)$ and the two-dimensional $(l=2)$ optimization have been implemented. For the one-dimensional case a golden section search is applied. The two-dimensional minimization makes use of the downhill simplex method [5].

\section{APPLICATION TO THE TESLA TEST FACILITY (TTF)}

The ALIGNMENT UTILITY was utilized for adjustment studies of the TTF laser driven electron-gun. The gun section consists of a 1.5 cell rf-cavity operating at $1.3 \mathrm{GHz}$, a cathode system, a bucking coil and two focusing solenoids (SOL1 and SOL2). For diagnostic purposes a beam position monitor (BPM1) is mounted at the gun exit. In our experiment we investigated the dependence of the beam spot position at BPM1 from the coil current of SOL1 varied in a range of $0 \ldots 400 \mathrm{~A}$ while bucking coil and SOL2 were kept switched off. In case of perfect alignment, i.e. a coincidence of cavity axis, magnetic axis of the solenoid and laser position on the cathode, a variation of the solenoid current does not have any influence on the beam position. Only if there is a shift between these axes the beam starts to move on a helix-like trajectory, the circular frequency $\omega_{H}$ of which, given by $\omega_{H}=e H / m c \gamma$, depends on the magnetic field $H$ and thus on the solenoid current [6]. An increase of the revolution frequency caused by a higher magnetic field involves that the electrons cover a larger fraction of the first helix-winding. The measurement therefore represents a projection of the electron path on the $x-y-p l a n e$ for the highest magnetic field set during the experiment.

The result of such a measurement is shown in figure 1 (red rhombs). It is compared with the values calculated by the Alignment Utility after each of four iterations in total. One iteration consists of a 2D optimization of the $\mathrm{x}$ and y-position of the laser beam spot on the cathode and a subsequent $2 \mathrm{D}$ optimization of the corresponding positions of the centre of SOL1. In iteration 1 and 2 BPM1 was assumed to be on axis. In iteration 3 and 4 a possible misalignment of BPM1 was taken into account by carrying out an additional analytical minimization. Even without considering an BPM1 offset the simulation qualitatively reproduces the movement of the beam spot on the $\mathrm{x}$-y-plane. However, there is a distinct deviation for low solenoid currents. Permitting a BPM1 offset the simulation yields a much more convincing agreement between the calculated and measured curve. Figure 2 demonstrates the development of the laser and solenoid position in the course of the four iterations. As iteration 4 does not yield a significant change compared to iteration 3 an additional optimization is not indicated. Table 1 summarizes the alignment parameter found after the last iteration. The given values may serve as instruction for adjusting solenoid and laser. By an alternating procedure of measuring and adjusting a remarkable improvement of the beam-line alignment could be achieved [6].
Table 1: Misalignments from simulation.

\begin{tabular}{|c|c|c|c|c|c|}
\hline \multicolumn{2}{|c|}{ laser beam } & \multicolumn{2}{c|}{ SOL1 } & \multicolumn{2}{c|}{ BPM1 } \\
\hline $\mathrm{x} / \mathrm{mm}$ & $\mathrm{y} / \mathrm{mm}$ & $\mathrm{x} / \mathrm{mm}$ & $\mathrm{y} / \mathrm{mm}$ & $\mathrm{x} / \mathrm{mm}$ & $\mathrm{y} / \mathrm{mm}$ \\
\hline-1.15 & +0.02 & -0.21 & +1.12 & 0.00 & +0.13 \\
\hline
\end{tabular}

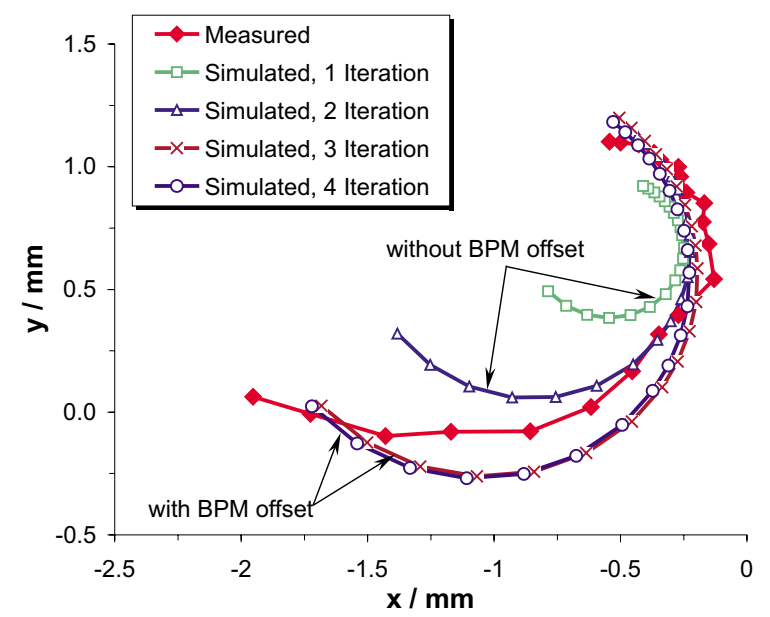

Figure 1: Comparison of measured and simulated beam positions at the gun exit after each iteration.

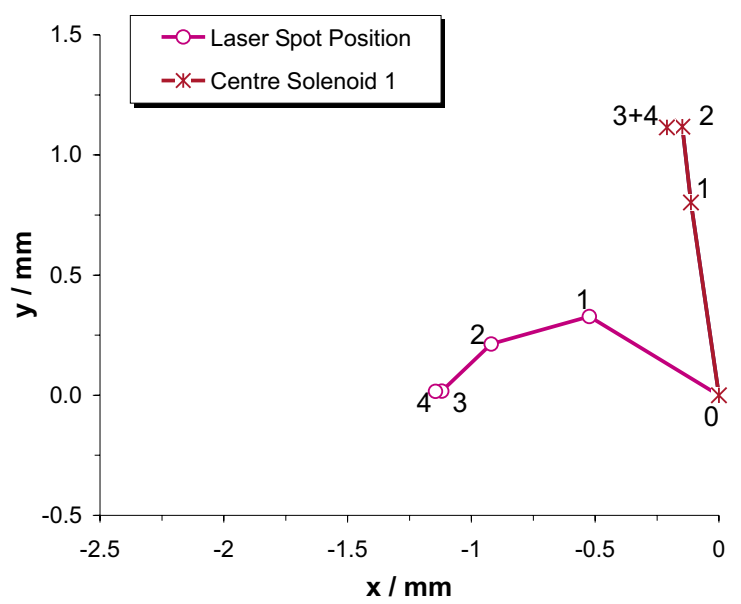

Figure 2: Laser spot and solenoid centre position after each iteration (indicated by the numbers).

\section{REFERENCES}

[1] M. Krassilnikov, A. Novokhatski, B. Schillinger, S. Setzer, T. Weiland, W. Koch, P.Castro, "V-Code Beam Dynamics Simulation", ICAP2000, Darmstadt, 2000.

[2] A. Novokhatski, T. Weiland, "Self-Consistent Model for the Beams in Accelerators”, ICAP'98, Monterey, 1998.

[3] A. Novokhatski, T. Weiland, M. Krassilnikov, W. Koch, P. Castro, "Beam Dynamics On-Line Simulation", LINAC2000, Monterey, 2000.

[4] A. Novokhatski, T.Weiland, "The Model of Ensembles for the Beam Dynamic Simulation”, PAC'99, New York, 1999.

[5] J.A. Nelder, R. Mead, Computer Journal, vol. 7, 1965.

[6] M. Krassilnikov, W. Beinhauer, P. Castro, R. Cee, W. Koch, A. Novokhatski, S. Ratschow, S. Schreiber, T. Weiland, "Beam-Based Alignment of TTF RF-Gun Using V-Code", PAC2001, Chicago, 2001. 